\section{Fabio Antonaci \\ Pierluigi Chimento \\ Hans-Christoph Diener \\ Grazia Sances \\ Giorgio Bono}

\title{
Lessons from placebo effects in migraine treatment
}

\section{Received: 3 November 2006 \\ Accepted in revised form: 8 January 2007 \\ Published online: 19 February 2007}

F. Antonaci • P. Chimento • G. Bono University Centre for Adaptive Disorders and Headache (UCADH),

Section of Varese, Italy

F. Antonaci (更) • P. Chimento • G. Sances Headache Centre,

IRCCS "C. Mondino Institute of

Neurology" Foundation,

Via Mondino 2, I-27100 Pavia, Italy

e-mail: neuronet@ libero.it

Tel.: +39-0382-3801

Fax: +39-02-700445466

H.-C. Diener

University of Duisburg,

Essen, Germany

\begin{abstract}
In medical research, the placebo effect is an important methodological tool. Placebo is given to participants in clinical trials, with the intention of mimicking an experimental intervention. The "nocebo" effect, on the other hand, is the phenomenon whereby a patient who believes that a treatment will cause harm actually does experience adverse effects. The placebo effect strongly influences the way the results of clinical trials are interpreted. Placebo responses vary with the choice of study design, the choice of primary outcome measure, the characteristics of the patients and
\end{abstract}

the cultural setting in which the trial is conducted. In migraine trials, the placebo response is high, in terms of both efficacy and side effects. Although medical ethics committees are becoming increasingly resistant to the use of placebo in acute migraine trials, placebo nevertheless remains the pivotal comparator in trials of migraine medications.

Keywords Placebo • Migraine • Triptans $\cdot$ NSAIDs $\cdot$ Nocebo

\section{Introduction}

The word "placebo", which literally means "I shall please", derives from the Latin Placebo domino in regione vivorum ("I shall please the Lord in the land of the living"). It was first used in the 14th century in reference to hired professional mourners at funerals and thus had associations with the ideas of depreciation and substitution [1]. Around the same time, Chaucer in The Canterbury Tales (The Merchant's Tale) depicts a wicked, parasitic and sycophantic character, whom he calls Placebo [2]. Much later, placebo came to mean a substance that can be given to humour or gratify a patient rather than to exert a genuine pharmacological effect.
The first recorded medical dictionary definition of placebo refers to "a commonplace method or medicine", commonplace meaning common and pedestrian. Indeed, in Motherby's 1795 dictionary, placebo was defined as "a commonplace method or medicine calculated to amuse for a time, rather than for any other purpose" [3]. This definition was maintained until 1937, when Taber's Digest of Medical Terms [4] defined placebo as an "inactive substance" and a "substitute for medicine given to deceive the patient".

Although a placebo is still regarded as an "inactive" substance, its impact can be profound. In clinical trials, if its effects are not measured, the placebo response may obscure a true pharmacological effect of an active comparator. 


\section{Psychological mechanisms}

There are at least four psychological mechanisms associated with the placebo response: (1) expectation, (2) conditioning, (3) therapeutic relationship and (4) empowerment [5]. Patients' expectations are based on their cultural background, on information given by the physician (dosage schedule, careful explanation of advantages and disadvantages) or written instructions, and on physical aspects of the placebo (colour, shape, taste, formulation). The old conditioning effect discovered by Pavlov is connected with displaying of the treatment: if symptom relief occurs following the administration of a particular tablet, the relief will, over time, become conditioned to occur following use of that particular tablet. There is also considerable evidence that a patient's interpersonal relationships play an important role. Social support influences a range of physiological parameters (heart, glands, immune system) and it provides a buffer against stress. Therefore, a patient-centred physician-patient relationship (e.g., proper interview conducted in a warm and friendly manner) is likely to produce a better outcome than a formal consultation. Last but not least, the placebo response is associated with empowerment, that is, the process of encouraging the patient to take an active part in the decision-making process regarding his treatment.

As all these factors can influence, to different extents in different patients, the size, variability and duration of a placebo response, this response becomes difficult to predict in advance.

\section{Neurobiological mechanisms}

Recently, there has been renewed scientific and public interest in the placebo effect following the publication of studies of its biological substrates. A placebo, through raised expectations and/or conditioning, can reduce pain by both opioid and non-opioid mechanisms. In the first case, placebo analgesia is typically blocked by the opioid antagonist naloxone, whereas in the second case it is not [6]. Central respiratory structures in the brainstem may also be inhibited by endogenous opioids as well as by placebo. The beta-adrenergic sympathetic system of the heart may also be inhibited during placebo analgesia, although the underlying mechanism is not known (reduction of the pain itself and/or direct action of endogenous opioids). Cholecystokinin [7] antagonises the effects of endogenous opioids, thereby reducing the placebo response. Placebo can also act on 5-HT-dependent hormone secretion, at both pituitary and adrenal gland level, thereby mimicking the effect of triptans.
There is evidence that the endogenous opioid system is implicated in the mediation of placebo effects under conditions of expectation of analgesia [8]. Using functional magnetic resonance imaging to measure, indirectly, neuronal activity during the administration of placebo with expectation of analgesia, Wager et al. [9] showed a significant effect on the activation of the $\mu$-opioid system (dorsolateral prefrontal cortex, rostral anterior cingulate, left nucleus accumbens and right anterior insula). PET studies have shown that in painful conditions placebo will activate the same central structures of the pain matrix as opioids [10].

\section{Placebo and headache}

According to the International Headache Society (IHS) [11], controlled trials of acute migraine medication should be carried out "in accordance with the principle of the Declaration of Helsinki" [12]. Later on, however, the same IHS guidelines explicitly recommend the use of placebo in trials of abortive medications. Therefore, a contradiction arises, as the declaration of Helsinki states that when an effective treatment for a disease exists, it is unethical to assign patients in a research study to a treatment known to be less effective. But standards for the acceptable use of placebo in clinical trials are changing. It is now necessary to provide ethical and scientific justification for the use of placebo in some therapeutic areas. The use of placebo in trials of acute medication is generally regarded as justified in situations in which withholding the best current treatment will result in only temporary discomfort and no serious adverse effects. In addition, most protocols allow the use of rescue medication two hours after intake of study medication.

\section{Placebo and migraine}

It is well known that the placebo response in clinical trials of acute migraine treatments is widely variable, ranging from $6 \%$ to $47 \%$ of patients. It has thus been recommended that active drugs for migraine must be shown to be significantly superior to placebo [12].

Bendtsen et al. [13] evaluated the placebo response in placebo-controlled randomised clinical trials of analgesics in migraine attacks that fulfilled the IHS criteria. Eleven studies were included in their review. A "headache response" (i.e., defined as a proportion of attacks that decreased in pain severity from moderate-severe to mild or no headache within $2 \mathrm{~h}$ ) was obtained after treatment 
with placebo in $30 \%$ (mean) of migraineurs, although the range (variability) was large $(7 \%-50 \%)$. Pain-free rates two hours after treatment with placebo were lower (mean $9 \%$, variability $7 \%-17 \%$ ), suggesting that that is a more robust outcome measure.

Loder et al. [14] reviewed literature reports (1991-2002) of placebo-controlled trials with triptans in acute migraine, and found 31 trials that met their inclusion criteria. The mean proportion of patients with a headache response to placebo at $2 \mathrm{~h}$ was $28.5 \pm 8.7 \%$ (range $15 \%-50 \%$ ), while the mean proportion of patients with a pain-free response to placebo at $2 \mathrm{~h}$ was $6.1 \pm 4.4 \%$ (range $5 \%-17 \%$ ).

Placebo response in children and adolescents with migraine presents a particular challenge, not least because placebo rates are enhanced in this age group [14]. Lewis et al. [15] reviewed the limited available data on the use of analgesics and triptans in placebo-controlled trials of migraine in children and adolescents and found a large variability in the placebo response: present in $37 \%-53 \%$ of patients treated with placebo analgesics/ non-steroidal anti-inflammatory drugs (NSAIDs), and in $28 \%-65 \%$ of those treated with placebo triptans. This explains why most trials investigating the efficacy of triptans in children and adolescents have failed to obtain conclusive findings [16]. As the response rates to triptans, however, were comparable with those found in adults, the variability of the placebo response must be taken into account, as must strategies to minimise it. One possibility could be to treat consecutive attacks with a placebo, which will likely show a decreasing response over time [17].

\section{The "nocebo effect"}

Although placebo response is used as an efficacy outcome, placebo is also associated with a spectrum of adverse events, reported across placebo-controlled clinical trials - the "nocebo" effect [18].

In a review of 109 double-blind, placebo-controlled drug trials involving a total of 1228 healthy volunteers, adverse effects were spontaneously reported by an average of $19 \%$ of those on placebo [19].

The following are examples of adverse effects that have been reported in patients receiving placebo, in placebo-controlled trials (any indication): drowsiness, fatigue, unsteady gait, mental confusion, motor retardation, insomnia, nervousness, motor agitation, headache, nausea, vomiting, constipation, diarrhoea, vertigo, dry mouth, leucopenia and death. In view of this, there is clearly a need to attempt to disentangle adverse effects associated with placebo from those associated with active medications, in order to arrive at a more accurate profile of the tolerability and safety of the active medication. In placebo-controlled trials of migraine, adverse effects may occur with placebo in $>30 \%$ of patients [19], and in triptan trials the mean $( \pm \mathrm{SD})$ proportion of patients reporting an $\mathrm{AE}$ on placebo was $23.4 \pm 14.1 \%$ (range 5\%-74\%) [20].

As placebo response rates in clinical trials in migraine may vary with different study designs, primary outcome measures and patient characteristics, and considering that the magnitude of the placebo effect can be influenced by a patient's expectations (conditioning), the severity of the pain at baseline and the rate of spontaneous resolution, placebo-subtracted outcome data may provide a more accurate profile of the active medication [13]. Adverse events with placebo triptans in migraine tend to be lower in European compared to North American studies [13], while within Europe, placebo response rates are higher in southern countries than in Scandinavia.

Even so, there are, inevitably, exceptional cases, as shown by the interesting report by Pascual-Lozano et al. [21], of a patient whose migraine, repeatedly refractory to analgesics, NSAIDs, ergotamine and opioids, responded well and consistently to placebo.

\section{Potential lessons to be learned from placebo-controlled trials in migraine}

Placebo response rates vary in studies presenting different designs, primary outcome measures and patient characteristics [12, 13]. However, the magnitude of these placebo effects in relation to active treatment does not justify the rate of spontaneous resolution, patient expectations (conditioning) and the severity of pain at baseline [12]. Furthermore, the lack of difference between a standard and a novel comparator does not prove the efficacy of the latter in the absence of a placebo group [10-12]. In addition, adverse events can only be appreciated when a placebo group is present. It has been shown that even though active drugs may be effective, a high placebo response rate may confound the evidence [18], and a high placebo response rate has been found to correlate with a high response on the corresponding active drug [12]. At the present time, comparison of results across trials is dangerous [12]. Placebosubtracted data enable a more rigorous comparison across different trials.

In summary, although medical ethics committees are becoming increasingly resistant to the use of placebo in acute migraine trials, placebo remains the pivotal comparator in trials of migraine medications. 


\section{References}

1. Shapiro AK (1964) A historic and heuristic definition of the placebo. Psychiatry 27:52-58

2. de Craen AJM (1999) Placebos and placebo effects in medicine: historical overview. J R Soc Med 92:511-515

3. Motherby G (1795) A new medical dictionary or general repository of physics, 4th edn. J. Johnson, London

4. Taber CW (1937) Taber's digest of medical terms. F.A. Davis, Philadelphia

5. Hyland ME (2003) Using the placebo response in clinical practice. Clin Med 3:347-350

6. Colloca L, Benedetti F (2005) Placebos and painkillers: is mind as real as matter? Nat Rev Neurosci 6:545-552

7. Benedetti F (1997) Cholecystokinin type-A and type-B receptors and their modulation of opioid analgesia. News Physiol Sci 12:263-268

8. Benedetti F, Mayberg HS, Wager TD et al (2005) Neurobiological mechanisms of the placebo effect. J Neurosci 25:10390-10402

9. Wager TD, Rilling JK, Smith EE et al (2004) Placebo-induced changes in fMRI in the anticipation and experience of pain. Science 303:1162-1167
10. Petrovic P, Kalso E, Petersson KM, Ingvar M (2002) Placebo and opioid analgesia - imaging a shared neuronal network. Science 295:1737-1740

11. International Headache Society Clinical Trials Subcommittee (2000) Guidelines for controlled trials of drugs in migraine. 2nd edn. Cephalalgia 20:765-786

12. World Medical Association (2002) World Medical Association Declaration of Helsinki: ethical principles for medical research involving human subjects. J Postgrad Med 48:206-208

13. Bendtsen L, Mattsson P, Zwart JA, Lipton RB (2003) Placebo response in clinical randomized trials of analgesics in migraine. Cephalalgia 23:487-490

14. Loder E, Goldstein R, Biondi D (2005) Placebo effects in oral triptan trials: the scientific and ethical rationale for continued use of placebo controls. Cephalalgia 25:124-131

15. Lewis DW, Winner P, Wasiewski W (2005) The placebo responder rate in children and adolescents. Headache 45:232-239
16. Lewis D, Ashwal S, Hershey A, et al (2004) Practice parameter: pharmacological treatment of migraine headache in children and adolescents: report of the American Academy of Neurology Quality Standards Subcommittee and the Practice Committee of the Child Neurology Society. Neurology 63:2215-2224

17. Diener HC (2003) Placebo in headache trials. Cephalalgia 23:485-486

18. Solomon S (2002) A review of mechanisms of response to pain therapy. Why voodoo works. Headache 42:656-662

19. Rosenzweig P, Brohier S, Zipfel A (1993) The placebo effects on healthy volunteers: influence of experimental conditions on the adverse events profile during phase I studies. Clin Pharmacol Ther 54:579-583

20. Ferrari MD, Goadsby PJ, Roon KI, Lipton RB (2002) Triptans (serotonin, 5-HT1B/1D agonists) in migraine: detailed results and methods of a metaanalysis of 53 trials. Cephalalgia 22:633-658. Erratum in: Cephalalgia (2003) $23: 71$

21. Pascual-Lozano AM, Chamarro-Lazaro R, Lainez MJ (2005) Placebo response in a patient with chronic migraine and ergotic overuse. Cephalalgia 25:391-394 